\title{
Far-Infrared Near-Field Optical Imaging and Kelvin Probe Force Microscopy of Laser- Crystallized and -Amorphized Phase Change Material Ge3Sb2Te6
}

Authors:

Julian Barnett ${ }^{*}+$, Lukas Wehmeier ${ }^{2,3}+$, Andreas Heßler ${ }^{l}$, Martin Lewin ${ }^{1}$, Julian Pries ${ }^{1}$, Matthias Wuttig ${ }^{1}$, J. Michael Klopf $f^{4}$, Susanne C. Kehr' ${ }^{2}$,Lukas M. Eng ${ }^{2,3}$, and Thomas Taubner ${ }^{1}$

Affiliations:

${ }^{1}$ I. Institute of Physics (IA), RWTH Aachen, 52074 Aachen, Germany

${ }^{2}$ Institute of Applied Physics, Technische Universität Dresden, 01062 Dresden, Germany

${ }^{3}$ ct.qmat, Dresden-Würzburg Cluster of Excellence-EXC 2147, Technische Universität

Dresden, 01062 Dresden, Germany

${ }^{4}$ Institute of Radiation Physics, Helmholtz-Zentrum Dresden-Rossendorf, 01328 Dresden, Germany

*Corresponding author: barnett@physik.rwth-aachen.de

${ }^{\dagger}$ J.B. and L.W. contributed equally to this paper.

\section{Supporting Information:}

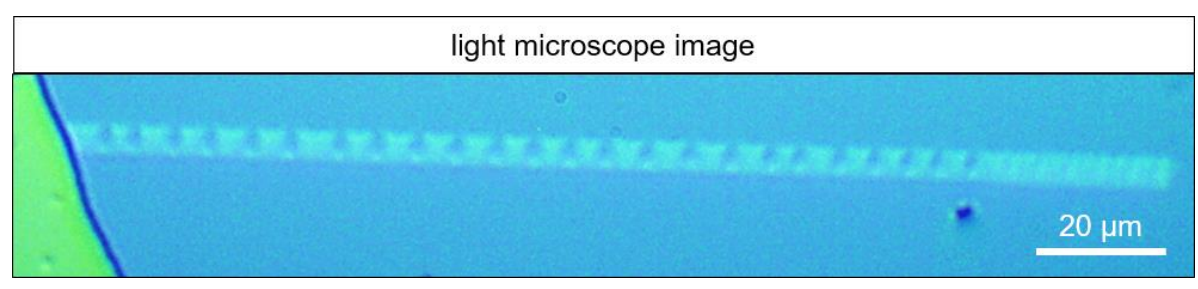

Figure S1. Overview of the sample layout: light microscope image of an as-deposited amorphous (A) GST326 sample (blue background) with gold edge (left) and laser-crystallized line ( $\mathbf{C}$, brighter blue) with reamorphized (RA) spots. The six spots at the end of the $\mathbf{C}$ line were recrystallized (RC) after their reamorphization.

\section{Methods}

Sputtering: A LS 320 von Ardenne system was operated in constant power mode $(20 \mathrm{~W})$ using stoichiometric targets of $99.99 \%$ purity. A layer of $150 \mathrm{~nm}$ nominal thickness of the phasechange material (PCM) $\mathrm{Ge}_{3} \mathrm{Sb}_{2} \mathrm{Te}_{6}$ (GST326) was deposited (background pressure $2 \cdot 10^{-6} \mathrm{mbar}$, $20 \mathrm{sccm}$ Ar flow, deposition rates $1.8 \AA$ ) on a highly doped Silicon substrate $(0.5 \mathrm{~mm}$ thickness, $<100>$ orientation, p-type doped with boron, 1-30 $\Omega \mathrm{cm}$ ) by direct current magnetron sputtering.

Optical switching: The localized phase-change (cf. section 2 below) of the GST326 thin-film was realized with an in-house-built laser setup. ${ }^{1,2}$ A pulsed laser beam (wavelength $\lambda=660 \mathrm{~nm}$ ) is focused by a 10-fold objective with a numerical aperture of 0.5 onto the sample surface. The sample is placed on a Thorlabs NanoMax-TS (Max311/M) stage, which is movable in x-, yand z-direction and connected to a Thorlabs closed-loop piezo controller (BPC303). A custom program allows for the automated positioning of pulsed laser shots on the sample surface within $5 \mathrm{~nm}$ accuracy. By using long (1000 ns) and low-powered $(15.6 \mathrm{~mW})$ laser pulses, the PCM thin-film was locally crystallized by heating it above the glass transition temperature. The crystallized area has an elliptical shape (cf. Figure S2b), whose size can be tuned by changing the laser parameters (pulse power, pulse duration and number of pulses) ${ }^{1}$. Successive 
crystallization of neighboring, overlapping areas resulted in the crystalline line (Figure S2a-II). By using short $(28 \mathrm{~ns})$ and high-powered $(300 \mathrm{~mW})$ pulses, the crystalline PCM was melted locally by heating it above the melting temperature, after which a rapid cooling $\left(>10^{9} \mathrm{~K} / \mathrm{s}\right)$ yielded amorphization (Figure S2a-III). Thus, multiple crystalline areas along the optically crystallized lines were reamorphized, forming reamorphized inclusions in the crystalline lines. Some of the reamorphized areas at the end of the crystalline lines were then recrystallized (Figure S2a-IV) with the crystallization parameters above (1000 ns at $15.6 \mathrm{~mW}$ ).

Free-electron laser: The free-electron laser FELBE (Helmholtz-Zentrum DresdenRossendorf, Dresden, Germany) is a pulsed, narrow-band radiation source $(\Delta \lambda / \lambda \approx 1 \%)$ with a central wavelength tunable from 5-250 $\mu \mathrm{m}\left(1.2-60.0 \mathrm{THz}, 40-2000 \mathrm{~cm}^{-1}\right)$ at $13 \mathrm{MHz}$ repetition rate. $^{3}$ The high repetition rate allows for application of methods developed for continuous wave sources such as higher-harmonic demodulation in SNOM.

SNOM: We used platinum-iridium-coated Si-Cantilevers (NanoSensors) driven at the mechanical resonance frequency of $\Omega_{\text {tip }} \approx 160 \mathrm{kHz}$ with a tapping amplitude of $A \approx 80 \mathrm{~nm}$. Radiation is focused onto the tip and collected via a 2" gold mirror with 2" focal length $(N A=1 / \sqrt{2})$, illuminated by half via a geometric beam splitter, which allows simultaneous power measurement and subsequent normalization of the detected light to beam power. We employ a self-homodyne detection scheme, leading to a combined response $p_{n}=s_{n} \cdot \cos \phi_{n}$ of optical amplitude $s_{n}$ and phase $\phi_{n}{ }^{4,5}$ Here, the optical phase is relative to the phase of the background scattering, which is diffrent from homodyne detection, where the phase is measured relative to the phase of a reference beam. Far-field background in SNOM was suppressed via higher-harmonic demodulation at $n \Omega_{\text {tip. }}{ }^{4,6}$ For data evaluation of far-infrared measurements $n=1$ was used, due to better signal to noise ratio and sufficient background suppression (cf. SI section 3, below), while $n=2$ was used for mid-infrared measurements. $\mathrm{CO}_{2}$ laser wavelengths (mid-infrared) were detected with a mercury cadmium telluride (MCT) detector (Judson technologies); for FEL measurements (far-infrared) we used a liquid-helium-cooled galliumdoped germanium photoconductor (QMC instruments). The experimental setup was placed in a nitrogen-purged environment $\left(\geq 99 \% \mathrm{~N}_{2}\right)$.

KPFM: We apply frequency-modulated KPFM as described in references. ${ }^{7-9}$ In summary, a voltage $U$ is applied between tip and sample holder (cf. Figure 1c of the main text). $U$ consists of a constant component $U_{\mathrm{KPFM}}$ and a sinusoidally varying component $U_{\mathrm{AC}}$, i.e., $U=U_{\mathrm{KPFM}}+U_{\mathrm{AC}}$. Here, we chose $U_{\mathrm{AC}}$ with a modulation frequency of $\Omega_{\mathrm{KPFM}}=2.0 \mathrm{kHz}$ and a modulation amplitude of $0.6 \mathrm{~V}$. In addition to this applied voltage, an intrinsic electric potential difference $U_{\mathrm{EPD}}$ between tip and sample exists. $U_{\mathrm{EPD}}$ conveys properties of the sample such as surface charging or a different work function ${ }^{8}$ and leads to a total potential difference $U_{\text {tot }}$ between tip and sample of $U_{\text {tot }}=U_{\mathrm{KPFM}}+U_{\mathrm{AC}}-U_{\mathrm{EPD}}$. $U_{\text {tot }}$ induces an additional electric force $F_{\text {el }}$ between tip and sample with ${ }^{8}$ :

$$
F_{e l}=\frac{1}{2} \frac{d C}{d z} U_{t o t}^{2}
$$

with $\frac{d C}{d z}$ being the capacitance gradient of the tip-sample system. The electric force $F_{e l}$, or more specifically its gradient $\frac{d F_{e l}}{d z}$, induces sidebands to the mechanical modulation of the cantilever at frequencies $\Omega_{\text {tip }} \pm \Omega_{\mathrm{KPFM}}$. In frequency-modulated KPFM a lock-in amplifier is used to 
detect the amplitude of these sidebands and $U_{\mathrm{KPFM}}$ is chosen such that this amplitude is minimized $^{8}$. As can be seen from formula (1), $F_{e l}$ is minimized for $U_{\mathrm{KPFM}}=U_{\mathrm{EPD}}$. Thus, KPFM simultaneously measures and compensates for any electric potential differences between tip and sample ${ }^{7-9}$. Additionally, KPFM also minimizes electrostatic artifacts in SNOM $^{9}$.

\section{Sample fabrication and layout}

Figure S1 presents a light microscope image of the relevant sample region, showing a gold edge on the left and a straight line of laser-crystallized GST326 (C, lighter blue) in a thin film of asdeposited amorphous GST326 (A, darker blue). On this line, several spots were laseramorphized (RA, darker blue spots on the line) and the final six spots were then lasercrystallized again (RC, brighter blue spots on the line), barely resolved by the light microscope.
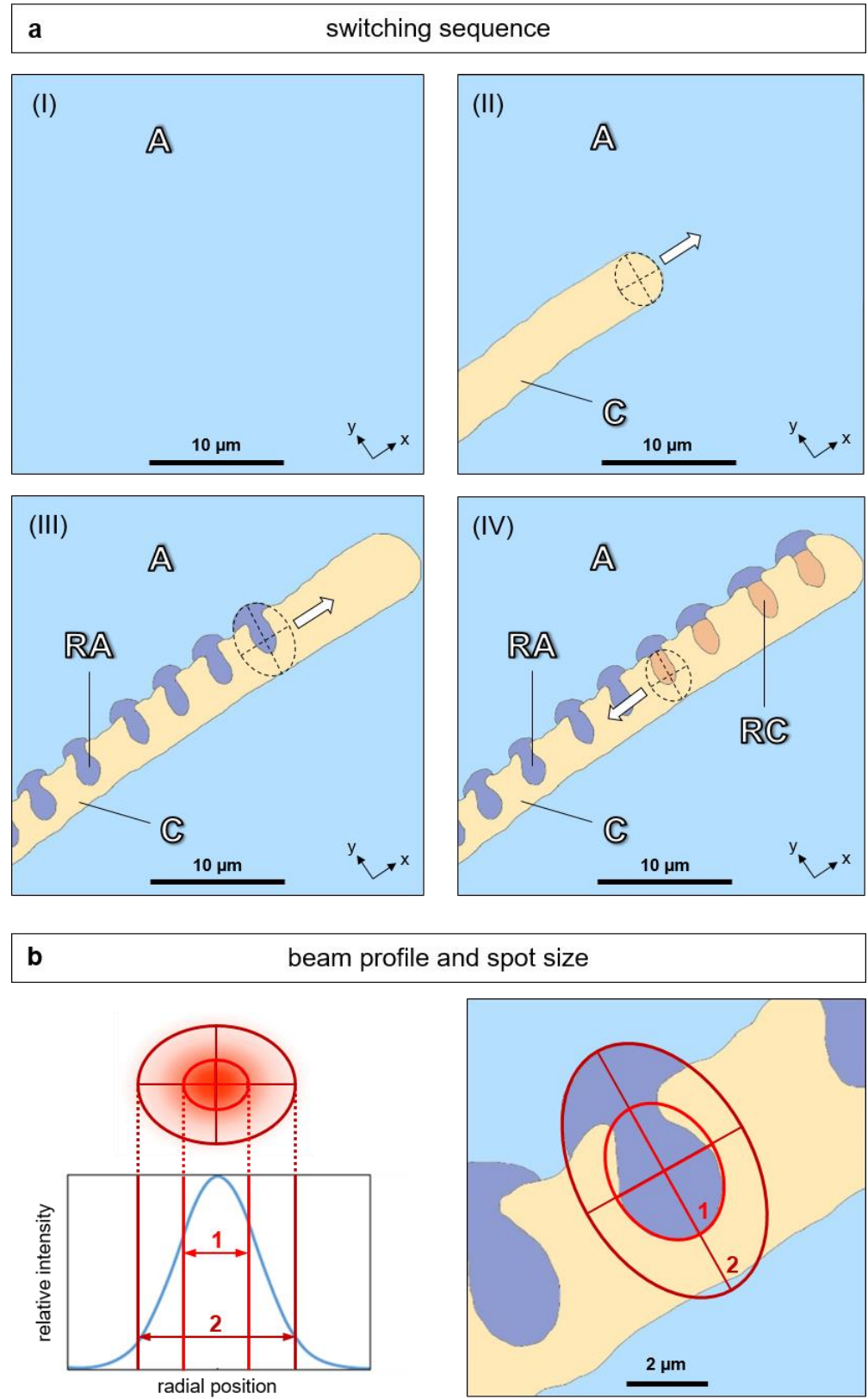

Figure S2. Details of the fabrication process. a) Schematic sketch of the laser switching sequence, depicting the A sample (I), the $\mathbf{C}$ line (II), the RA spots (III), and the RC spots (IV). Dashed black ovals mark the position of 
the laser beam for the crystallization (II), reamorphization (III), and recrystallization (IV) step, respectively, leading to a slight offset. White arrows indicate the moving direction of the laser spot. b) Schematics of the reamorphization process, showing the influence of the Gaussian intensity profile, with different intensity levels $\mathbf{1}$, $\mathbf{2}$ marked by vertical red lines in the beam profile and by red ovals in the top view. The mushroom shape of the RA spots results from different power thresholds for the switching process (see text for details).

Figure S2a shows the sequence of the switching steps, starting from the as-deposited amorphous sample A (I), crystallizing a $\mathbf{C}$ line consisting of overlapping spots (II), reamorphizing $\mathbf{R A}$ spots along the line (III), and, finally, recrystallizing six RC spots at the end of the line (IV). Further cycling between amorphous and crystalline states was not done in this work and will be investigated in future experiments. Additionally, Figure S2a shows the influence of positioning of the laser spots (dashed ellipses), as each step of the switching sequence can be offset slightly. To explain the mushroom shape of the resulting RA spots, Figure S2b presents the influence of the Gaussian intensity distribution of the switching laser, where different intensity levels are marked in the schematic line profile (vertical red lines $\mathbf{1}$ and 2) and presented as an overlay (red ovals $\mathbf{1}$ and 2) in the schematic of the RA spot. For reamorphization to happen, the PCM material has to be heated above the melting temperature and then quenched rapidly. In the laser switching process, the deposited energy is distributed inhomogeneously due to the Gaussian intensity profile. Additionally, the thermal conductivity of the sample (and indeed each layer, i.e. substrate, PCM layer, superstrate) strongly influences the resulting local temperature during the non-stationary process. When the laser spot is heating a material boundary, both effects (intensity profile and change in thermal conductivity) lead to a spatially non-uniform phase transition ${ }^{10}$. For the GST326 sample used in this work, the center position of the RA spot was slightly offset from the C line. Similarly to the properties of GST225 reported in literature ${ }^{10}$, we expect the thermal conductivity of crystalline GST326 to be higher than that of amorphous GST326. Therefore, a higher intensity ( $\mathbf{1}$ as opposed to $\mathbf{2}$ ) is necessary to reach temperatures above the melting point inside the $\mathbf{C}$ line, resulting in a smaller $\mathbf{R A}$ spot size compared to outside the $\mathbf{C}$ line, and therefore the mushroom shape.

\section{Comparison of the near-field signal in first and second order harmonic demodulation}

To suppress contributions to the scattered light that do not result from the near-field of the tip (i.e. background), the detector signal is demodulated at higher-harmonic frequencies $n \Omega_{\text {tip }}$ of the tip oscillation frequency $\Omega_{\text {tip. }}$. As the near-field coupling is strongly non-linear with the distance between tip and sample, its contribution to the signal is transferred to higher demodulation orders, while the more linear background is increasingly suppressed. For this reason, it is in principle desirable to use data from high demodulation orders. However, as the overall signal decreases with $n$, noise has to be taken into consideration and a balance between background suppression and signal strength has to be found.

The efficiency $\eta$ of background suppression depends on demodulation order $n$, tapping amplitude $A$, and wavelength $\lambda$, and can be approximated by $\eta \sim(\lambda / A)^{n} .4,11-13$ If the wavelength becomes very large in relation to the tapping amplitude, the background fields show only minimal variation on the length scale of the tip oscillation amplitude. This intuitively explains why background suppression is more effective for longer wavelengths and why demodulation orders of $n=3,4$ are necessary for visible or near-infrared wavelengths, while $n=2$ often suffices for mid-infrared measurements. In the far-infrared (THz) range, even $n=1$ yields nearfield data of good quality. ${ }^{12}$ 
To exclude measurement artefacts introduced by insufficiently suppressed background, Figure S3 qualitatively compares the near-field signal with demodulation at $n \Omega_{\text {tip }}$ for $n=1$ (top row) and $n=2$ (bottom row) for our far- and mid-infrared measurements. Besides the signalto-noise ratio, no significant deviations across the image are visible, justifying the use of $n=1$ in far-infrared measurements to extract the line profiles shown in Figure 3 in the main text.

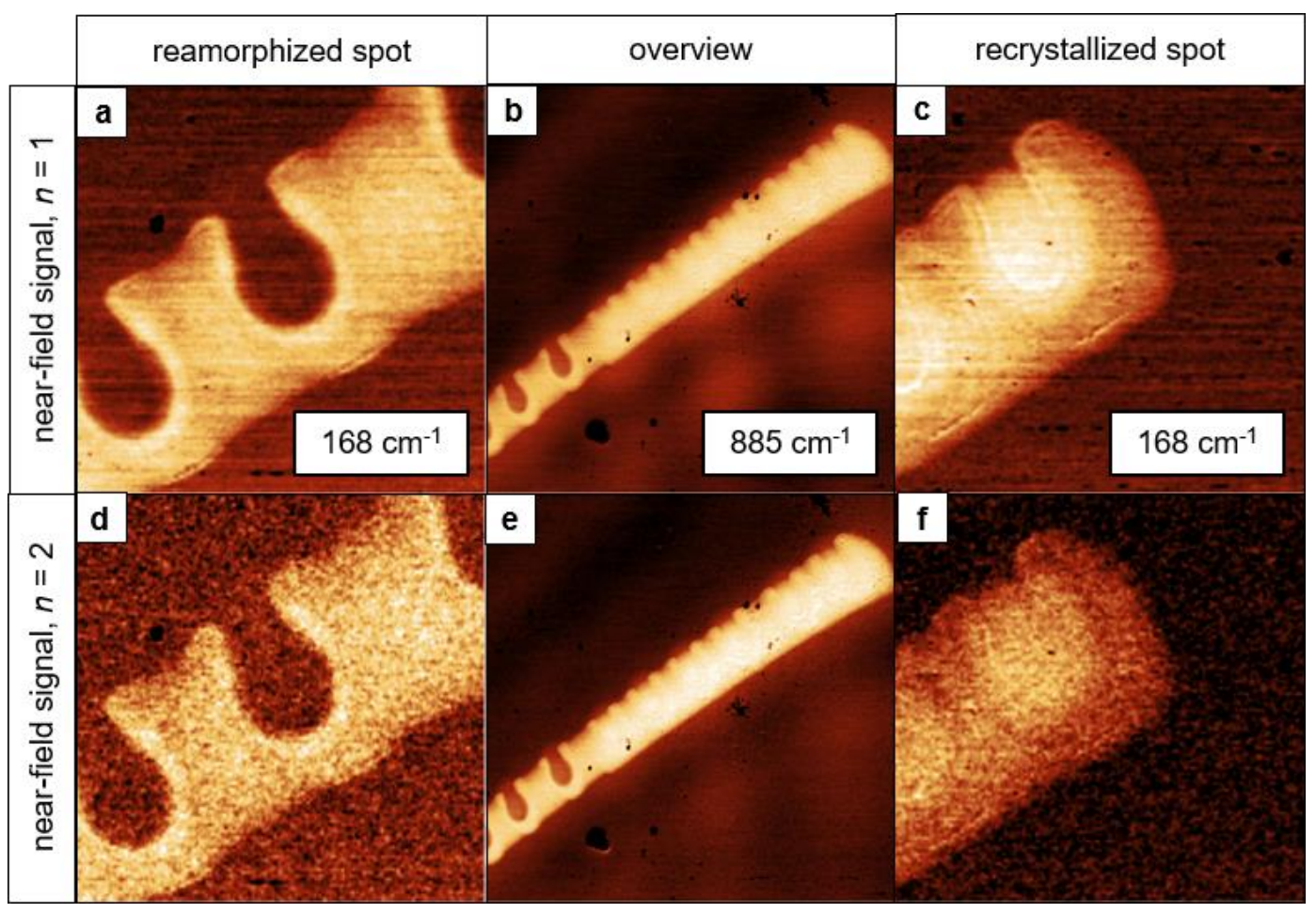

Figure S3. Qualitative comparison of the optical near-field signal with demodulation at $n \Omega_{\text {tip }}$ for $n=1$ (top row) and $n=2$ (bottom row). In the main text, demodulation with $n=1$ is displayed for far-infrared (Figures $2 \mathrm{~d}$ and $2 \mathrm{f}$ ) and $n=2$ for mid-infrared measurements (Figure 2e). The overview image (center) is $30 \mu \mathrm{m} \times 30 \mu \mathrm{m}$ in size and the zoom-ins (left, right) are $10 \mu \mathrm{m} \times 10 \mu \mathrm{m}$.

To enable a quantitative analysis of SNOM signals, the remaining additive background can be eliminated via self-referencing, i.e. the subtraction of a constant contribution. Here, the amorphous state $\mathbf{A}$ was used as a reference (new zero-level) to compare the frequencydependent change in SNOM contrast between RC and $\mathbf{C}$ states. As such, the SNOM contrast between $\mathbf{R C}$ and $\mathbf{C}$ state is defined as

$$
c_{\mathrm{SNOM}}^{\mathrm{RC}, \mathrm{C}}=\frac{\left(p_{1}^{\mathrm{RC}}-p_{1}^{\mathrm{A}}\right)}{\left(p_{1}^{\mathrm{C}}-p_{1}^{\mathrm{A}}\right)},
$$

i.e. the ratio between the self-referenced SNOM signals $p_{n}$ (at $n=1$ ) of the $\mathbf{R C}$ and $\mathbf{C}$ state, each in relation to the amorphous state. This method allows for comparison to theoretical models (cf. Figure S5) and therefore for insight into the electronic properties of the two crystalline states. 


\section{Comparison of SNOM signal of crystalline states in mid- and far-infrared}
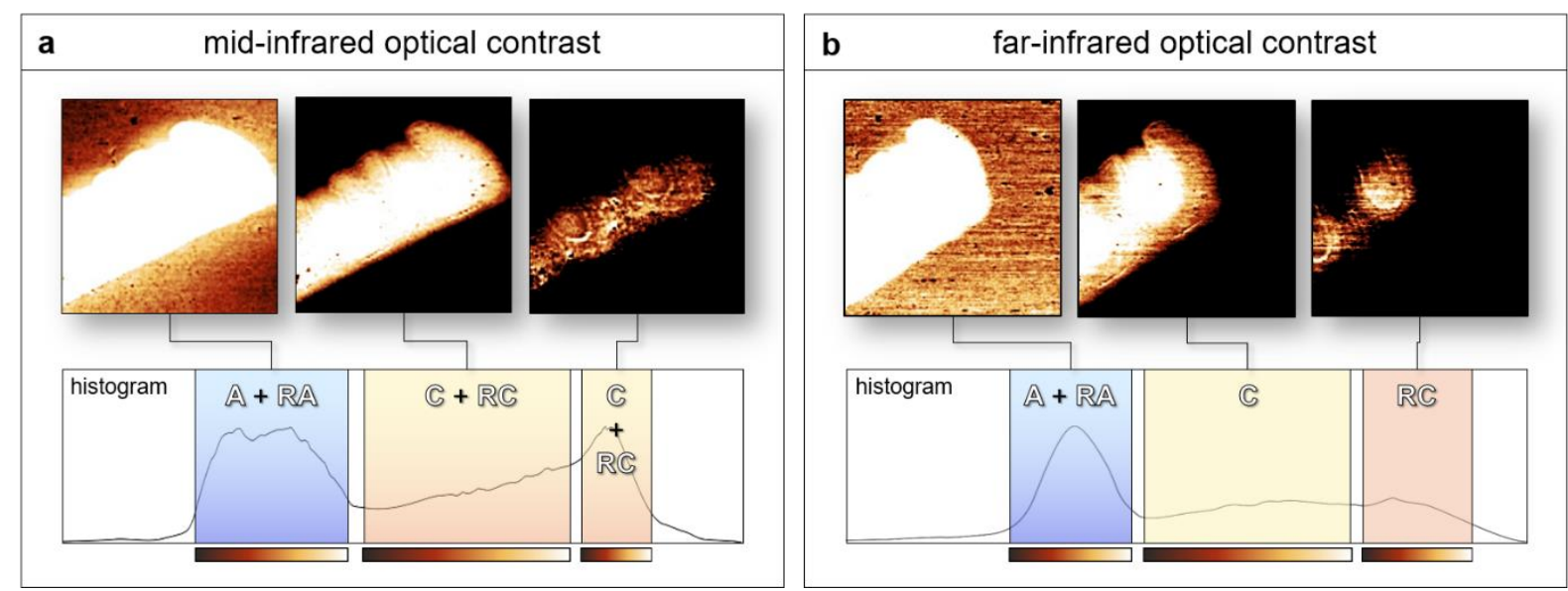

Figure S4 Qualitative comparison of mid-infrared $\left(885 \mathrm{~cm}^{-1}, \mathrm{CO}_{2}\right.$ laser $)$ and far-infrared $\left(168 \mathrm{~cm}^{-1}\right.$, FEL $)$ optical near-field signal $p_{1}$ of $\mathbf{R C}$ spots, comparing the data of Figures 2 and 4 of the main text in more detail. a) Bottom: histogram of the optical near-field signal in the mid-infrared $\left(885 \mathrm{~cm}^{-1}\right.$, cutout of Figure 2e). Top: near-field images $(10 \mu \mathrm{m} \times 10 \mu \mathrm{m}$ in size) with different ranges selected for the color scale (linked by black lines). The color ranges are marked by shaded regions in the histogram, corresponding to the different states: amorphous (blue) and crystalline (orange), cf. Figure S2. b) Same quantities as presented in (a) for the far-infrared $\left(168 \mathrm{~cm}^{-1}, \mathrm{cf}\right.$. Figure 2f), enabling separation of $\mathbf{C}$ (lighter shading in the histogram) and $\mathbf{R C}$ (darker shading) states.

Figure S4 analyzes the qualitative difference in contrast between the far- and mid-infrared measurement (Figure 4 of the main text) in more detail. In particular, the histograms of the optical signal are presented at the bottom, from which different images are extracted (top), when setting the color scale to a certain range (shaded regions). Notably, the $\mathbf{C}$ and $\mathbf{R C}$ areas are represented at different values in the histogram in the far-infrared (Figure S4b), while they are intermixed and indistinguishable in the mid-infrared (Figure S4a). As a result, far-infrared measurements can be used to differentiate between crystalline states, which appear identical in the mid-infrared (cf. contrast simulation in Figure S5).

\section{SNOM contrast dependence on free charge carrier concentration}

The SNOM contrast between $\mathbf{R C}$ and $\mathbf{C}$ state was defined in equation (2) as

$$
c_{\mathrm{SNOM}}^{\mathrm{RC}, \mathrm{C}}=\frac{\left(p_{1}^{\mathrm{RC}}-p_{1}^{\mathrm{A}}\right)}{\left(p_{1}^{\mathrm{C}}-p_{1}^{\mathrm{A}}\right)},
$$

i.e. the ratio between self-referenced SNOM signals $p_{1}$ of the $\mathbf{R C}$ and $\mathbf{C}$ state. This approach was chosen to eliminate potentially remaining additive background due to the low demodulation order (demodulation of $p_{n}$ at frequency $n \Omega_{\text {tip }}$ with $n=1$ ). Using this method, a contrast of $c_{\mathrm{SNOM}}^{\mathrm{RC}, \mathrm{C}}=1.8$ was determined in the far-infrared measurement, while no contrast $\left(c_{\mathrm{SNOM}}^{\mathrm{RC}, \mathrm{C}}=1\right)$ could be observed in the mid-infrared measurement. As a possible explanation, we propose a change in electronic properties (charge carrier concentration $n$, mobility $\mu$, or effective mass $m^{*}$ ) in the main text but the direct determination of quantitative values from SNOM measurements is difficult in practice. The reason for this is that both the relationship between SNOM signal and dielectric function, as well as the relationship between dielectric function and electronic properties, are non-bijective and strongly non-linear (cf. Figure S9). As such, 
measurements have to be compared to simulations to show that a proposed change in electronic properties can indeed explain the observed SNOM contrast.

In our theoretical modeling (see the following sections for details), amplitude $s_{n}$ and phase $\phi_{n}$ are determined separately, while they cannot be separated in self-homodyne measurements. However, we show that the influence of the phase is negligible in this case (see section 8 below), allowing us to compare our measured SNOM signals $p_{n}$ to simulated SNOM amplitudes $s_{n}$ :

$$
c_{\mathrm{SNOM}, \mathrm{sim}}^{\mathrm{RC}, \mathrm{C}}=\frac{\left(s_{1}^{\mathrm{RC}}-s_{1}^{\mathrm{A}}\right)}{\left(s_{1}^{\mathrm{C}}-s_{1}^{\mathrm{A}}\right)},
$$

As a result, Figure $\mathrm{S} 5$ shows the influence of rising $n$ on $c_{\mathrm{SNOM}, \mathrm{sim}}^{\mathrm{RC}, \mathrm{C}}$ (cf. Figure $\mathrm{S} 8$ for individual signals $s_{1}^{\mathrm{RC}}, s_{1}^{\mathrm{C}}$, and $s_{1}^{\mathrm{A}}$ ), where a charge carrier concentration of $n_{\mathrm{C}}=1 \times 10^{20} \mathrm{~cm}^{-3}$ was used for the $\mathbf{C}$ state, in accordance with literature values ${ }^{14}$. The rising charge carrier concentration has a much stronger influence in the far-infrared region than the mid-infrared region, matching our observation in measurements at frequencies of $168 \mathrm{~cm}^{-1}$ and $885 \mathrm{~cm}^{-1}$, respectively (marked by vertical grey lines, cf. Figure 4 of the main text). Here, a factor of $c_{\mathrm{SNOM}, \text { sim }}^{\mathrm{RC}, \mathrm{C}}=1.8$ can be achieved at $168 \mathrm{~cm}^{-1}$ by a change in $n$ of one order of magnitude $\left(n_{\mathrm{RC}}=1.1 \times 10^{21} \mathrm{~cm}^{-3}\right)$ in the RC state, while the contrast at $885 \mathrm{~cm}^{-1}$ remains close to unity. As this matches our experimental results very well, we conclude that a change in electronic properties is the most likely explanation for the observed contrast. However, as the disentanglement of electronic properties $\left(n, \mu, m^{*}\right)$ in the regime of high damping (i.e. low mobility) is difficult ${ }^{14}$, changes in charge carrier mobility and effective mass might also play a role, besides an increase in charge carrier concentration.

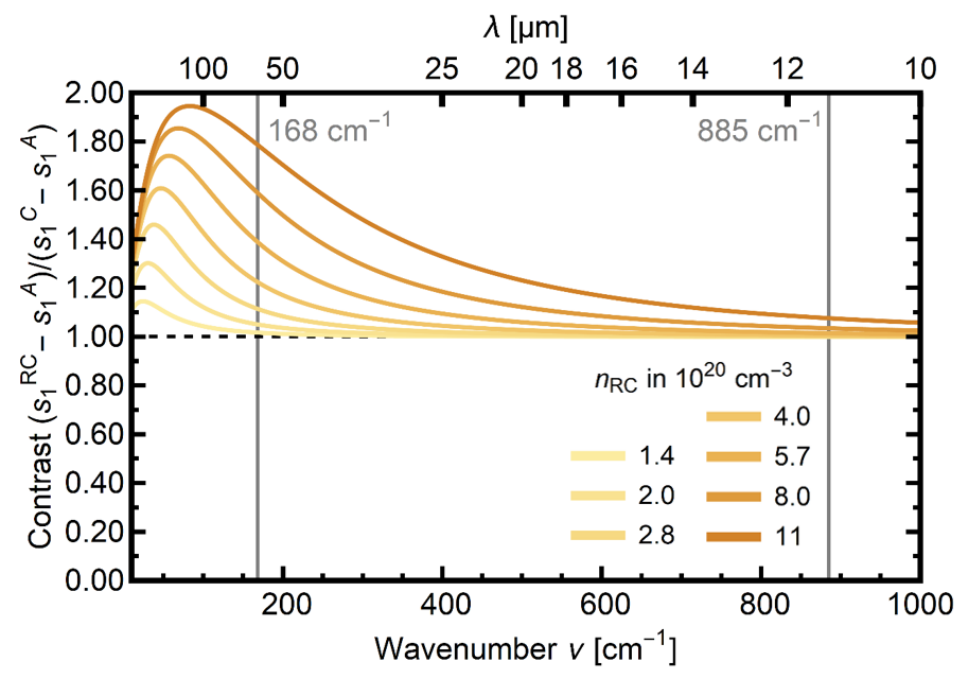

Figure S5. Dependence of simulated SNOM contrast $c_{\mathrm{SNOM}, \text { sim }}^{\mathrm{RC}, \mathrm{C}}$ on charge carrier concentration $n_{\mathrm{RC}}$ of the selfreferenced $\mathbf{R C}$ state, normalized to the self-referenced $\mathbf{C}$ state with $n_{\mathrm{C}}=1 \times 10^{20} \mathrm{~cm}^{-3}$, in accordance with literature values for GST326 $\left(\mu=0.9 \mathrm{~cm}^{2} / \mathrm{Vs}\right.$ and $\left.m^{*}=0.3 m_{0}\right){ }^{14}$ 


\section{Dielectric function of GST326 (far-field measurement)}

Figure S6 shows the real (solid) and imaginary (dashed) part of the far-field dielectric functions $\varepsilon$ of crystalline (yellow) and amorphous (blue) GST326 for the infrared and visible spectral range. It consists of a band gap absorption that follows the behavior of a Tauc-Lorentz oscillator (Figure S6a), with large $\operatorname{Re}[\varepsilon]$ below the band gap in the crystalline phase, which is a typical indicator of metavalent bonding ${ }^{15}$. The imaginary part of the crystalline phase, which is linked to the optical losses inside the material, is high above $0.5 \mathrm{eV}$ due to band gap absorption and strongly rises for $E \rightarrow 0 \mathrm{eV}$ due to Drude contributions of free charge carriers. In the midinfrared region (Figure S6b), optical losses are very low in both crystalline and amorphous phase, making GST326 ideally suited for low-loss mid-infrared nanophotonic applications. The shaded region up to $E \approx 0.25 \mathrm{eV}$ in Figure S6a indicates the frequency limit $\left(v=2000 \mathrm{~cm}^{-1}\right)$ of the zoom-in presented in Figure S6b, which shows the mid- and far-infrared range. Here, the measurement frequencies at $168 \mathrm{~cm}^{-1}$ and $885 \mathrm{~cm}^{-1}$ are indicated by vertical dotted lines.

a

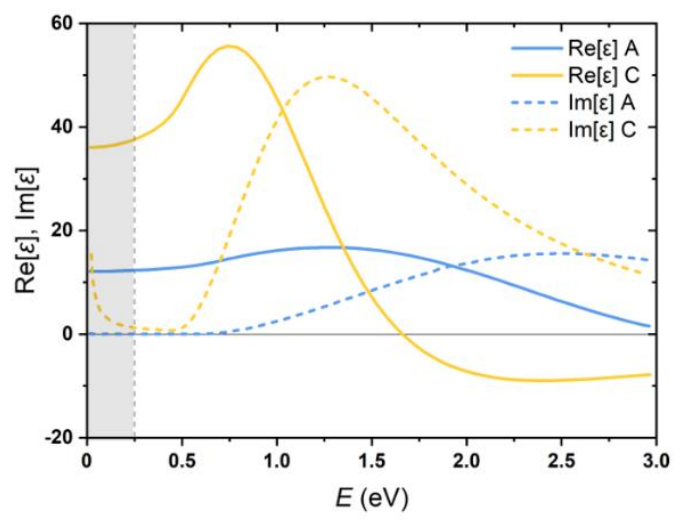

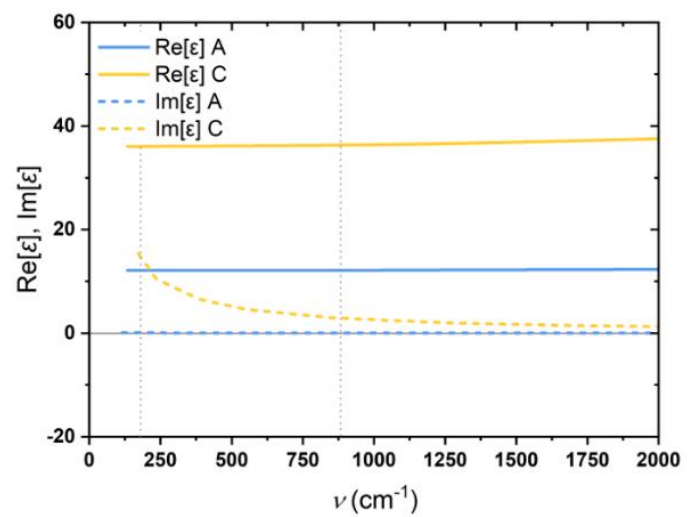

Figure S6. Dielectric function of GST $326^{16}$, a) showing the Tauc-Lorentz profile with absorption above the band gap of $E_{\mathrm{g}} \approx 0.5 \mathrm{eV}$ and a Drude term describing the free electron contribution. Real (solid lines) and imaginary parts (dashed lines) of both amorphous (A, blue) and crystalline (C, yellow) GST326 are shown. The shaded region up to the vertical dashed line at $E \approx 0.25 \mathrm{eV}$ indicates the range of $\mathbf{b}$ ), showing the far- to mid-infrared region of $0-2000 \mathrm{~cm}^{-1}$, with the measurement frequencies at $168 \mathrm{~cm}^{-1}$ and $885 \mathrm{~cm}^{-1}$ marked by vertical dotted lines. The real part of the crystalline state (solid yellow line) is increased by a factor of 3 compared to the amorphous state (solid blue line), which is an indicator of metavalent bonding ${ }^{15}$. The imaginary part of the crystalline state (dashed yellow line) shows an additional Drude contribution due to free charge carriers, which are not present in the amorphous state (dashed blue line). 


\section{Simulation of SNOM contrast}

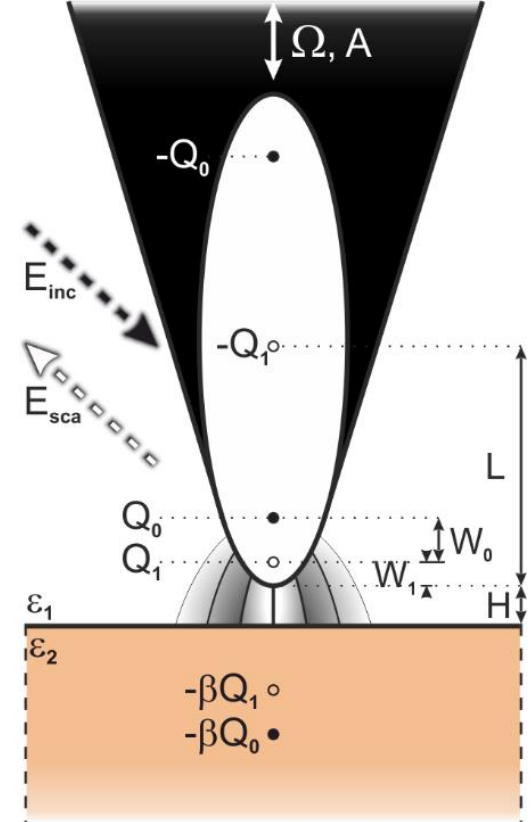

Figure S7. Schematic of the Finite Dipole Model.
To model the SNOM contrast, the Finite Dipole Model $(\mathrm{FDM}){ }^{17}$ was employed in combination with a Transfer Matrix Method (TMM), resulting in a powerful predictive tool to simulate the near-field response of arbitrarily layered systems with known dielectric function. ${ }^{18}$

The relevant FDM parameters (cf. Figure S7) used in these simulations are the length $L=300 \mathrm{~nm}$ of the ellipsoid, tip radius $R=W_{l}=30 \mathrm{~nm}$, distance $W_{0}=1.31 \cdot R$, tapping amplitude $A=75 \mathrm{~nm}$, geometric factor $g=0.7 \cdot \mathrm{e}^{0.06 \mathrm{i}}$, and demodulation order $n=1$. An explanation of the empirical parameter values can be found in literature. ${ }^{19}$

In contrast to the bulk model, ${ }^{17}$ here the near-field coupling term $\beta$ can be replaced by the reflection coefficient $r_{\mathrm{p}}$ at the dominating spatial frequency of $k_{\mathrm{x}}=250000 \mathrm{~cm}^{-1}$, as given by the inverse tip radius, instead of the electrostatic limit

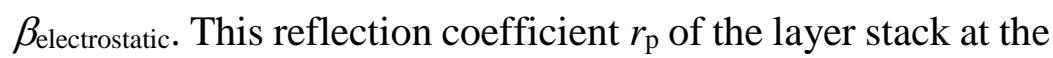
dominant $k_{\mathrm{x}}$ value is calculated from a transfer matrix that describes the sample composition using the permittivities $\varepsilon$ and thicknesses $d$ of all layers. ${ }^{20}$

\section{Validity of SNOM amplitude and phase in self-homodyne detection}

The self-homodyne detection scheme does not allow for the separation of SNOM amplitude $s_{1}$ and phase $\phi_{1}$, instead, the detected signal $p_{1}=s_{1} \cdot \cos \phi_{1}$ depends on both. As such, simulations of amplitude (Figure S8a) and phase (Figure S8b) were done to check whether a comparison with the detected signal is valid. Here, the change in phase between the different states is miniscule $\left(\Delta \phi_{1}<0.06 \mathrm{rad}\right)$, which amounts to changes of only $0.2 \%$ in the detected signal $p_{1}$, much smaller than variations introduced by the amplitude $s_{1}$. As such, a direct comparison between amplitude and detected signal is reasonable in this case.
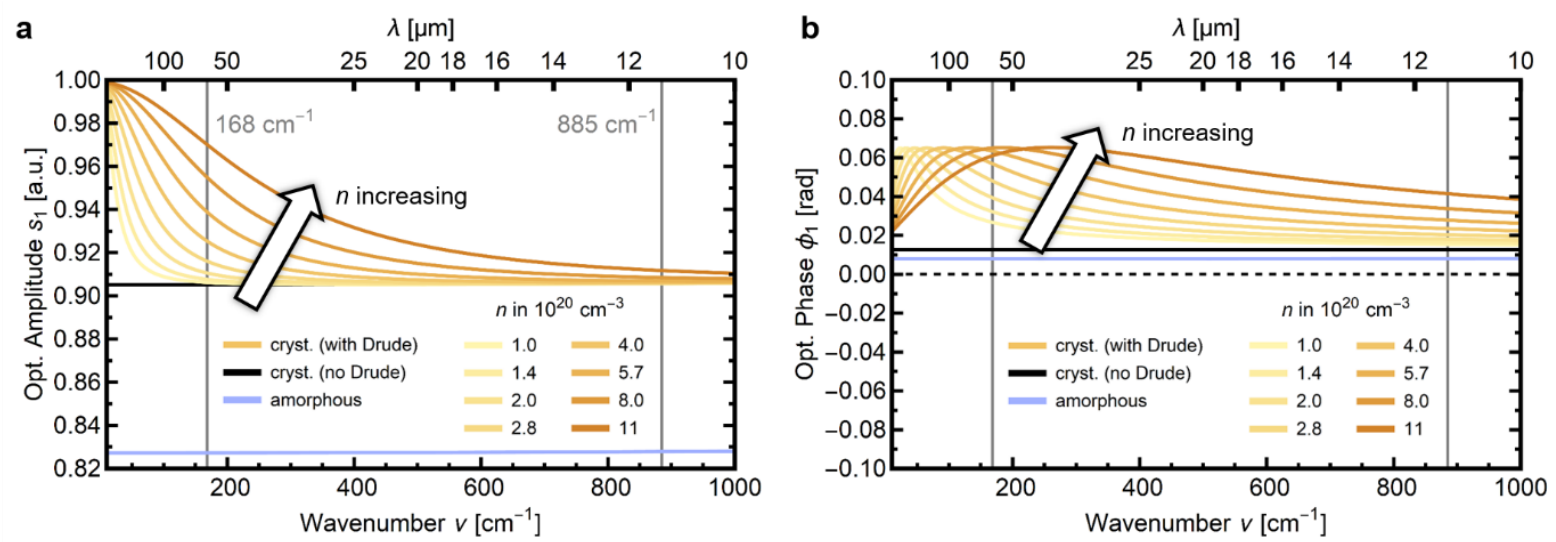

Figure S8. Near-field optical a) amplitude and b) phase of amorphous (light blue) and crystalline (black) GST326, the latter with different charge carrier concentration $n$ (yellow) between 1 and $11 \times 10^{20} \mathrm{~cm}^{-3}\left(\mu=0.9 \mathrm{~cm}^{2} / \mathrm{Vs}\right.$ and $\left.m^{*}=0.3 m_{0}\right)$. The arrows indicate the influence of rising $n$. 
Figure S8a also shows the calculated signal for each of the states that are necessary to calculate the contrast $c_{\mathrm{SNOM} \text {,sim }}^{\mathrm{RC}, \mathrm{C}}$ in Figure S5. The light blue (amorphous) curve illustrates the background that is subtracted in the self-referencing step (cf. section 3 above). The black (crystalline) curve represents the change in SNOM signal introduced by the change in refractive index typical for PCMs $^{15}$ (cf. Figure S6) via $\varepsilon_{\infty}$. Adding a Drude term to describe free charge carriers (cf. Figure S9a) leads to an increase in signal at lower frequencies (yellow $\rightarrow$ orange curves), with a higher charge carrier concentration $n$ shifting this contribution towards higher frequencies (arrows).

\section{SNOM signal of free electrons in the regime of high damping}

The contribution of free charge carriers to the dielectric function of GST326 in the infrared region can be described by the Drude model (Figure S9a). However, the damping factor $\gamma$ in GST326 is high enough to result in unusual behaviour. For low damping $\left(\gamma<<\omega_{\mathrm{p}}\right.$, where $\omega_{\mathrm{p}}$ denotes the plasma frequency of the free charge carriers), the real part of the dielectric function is dominated by the term $-\omega_{\mathrm{p}}^{2} / \omega^{2}$, diverging to $-\infty$ for $\omega \rightarrow 0$. However, in the case of GST326, the mobility is rather low $\left(\mu<1.1 \mathrm{~cm}^{2} / \mathrm{Vs}\right)^{14}$, leading to strong damping. In this case, $\gamma$ (marked blue in the formula in Figure S9a) is non-negligible compared to $\omega_{\mathrm{p}}$ and leads to a near-constant real part of the dielectric function (Figure S9b, blue curves). As the imaginary part has the additional factor of $\omega$ in the denominator (marked red in the formula in Figure S9a), it still diverges to $+\infty$ for $\omega \rightarrow 0$ (Figure S9b, red curves). Overall, an increase in $n$ (indicated by white arrows) has nearly negligible impact on the real part of the dielectric function, while it strongly influences the imaginary part. The unusually large contribution from the non-Drude background $\varepsilon_{\infty}$ is typical for metavalent bonding in crystalline $\mathrm{PCMs}^{15}$ and leads to a value of $\operatorname{Re}[\varepsilon] \approx 35$ in this case, which is important to note (cf. Figure S6). Finally, the vertical grey lines indicate the relevant measurement frequencies, that are discussed in the main text, showing that the difference in the imaginary part is much more pronounced in the far-infrared measurement at $v=168 \mathrm{~cm}^{-1}$, compared to the mid-infrared measurement at $v=885 \mathrm{~cm}^{-1}$.

Figures S9c and S9d show the predicted image contrast ("coupling function") 21,22 of the SNOM signal, calculated using the Finite Dipole Model. This coupling function describes how light is scattered from the coupled tip-sample system for a certain dielectric function of the (bulk) substrate. Consequently, the $x$-axis describes the real part of the dielectric function $\operatorname{Re}[\varepsilon]$, while the evolution of the curves (towards thicker lines) shows the influence of increasing imaginary part $\operatorname{Im}[\varepsilon]$ from 0.5 to 64 , which dampens the near-field resonance around $\operatorname{Re}[\varepsilon] \approx-1$, where tip and sample are strongly coupled. However, due to the large $\operatorname{Re}[\varepsilon]$ of GST326, our measurements are far away from the resonant case, as indicated by the dashed rectangles. Figures S9e and S9f show zoom-ins of this region around $\operatorname{Re}[\varepsilon] \approx 35$, with the influence of increasing $\operatorname{Im}[\varepsilon]$ from 0.5 to 64 , as is relevant in our measurements (cf. Figure $S 9 b$ ), indicated by white arrows. Contrary to the resonant case, here a rising imaginary part leads to an increase of SNOM signal in both amplitude and phase. 
$\mathbf{a}$

$$
\begin{aligned}
\varepsilon_{\mathrm{GST} 326} & =\varepsilon_{\infty}+\varepsilon_{\text {Drude }} \\
& =\varepsilon_{\infty}-\underbrace{\frac{\omega_{\mathrm{p}}^{2}}{\omega^{2}+\gamma^{2}}}_{\text {real part }}+\underbrace{\frac{\gamma \omega_{\mathrm{p}}^{2}}{\omega\left(\omega^{2}+\gamma^{2}\right)}}_{\text {imaginary part }}
\end{aligned}
$$

b

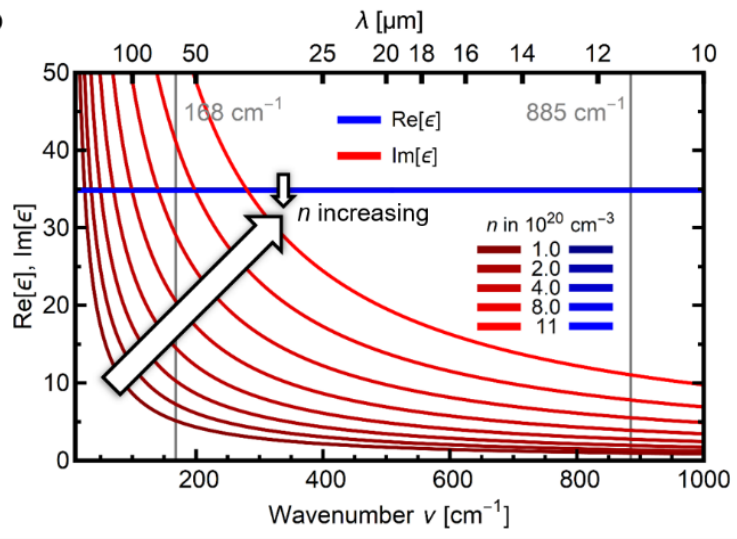

C

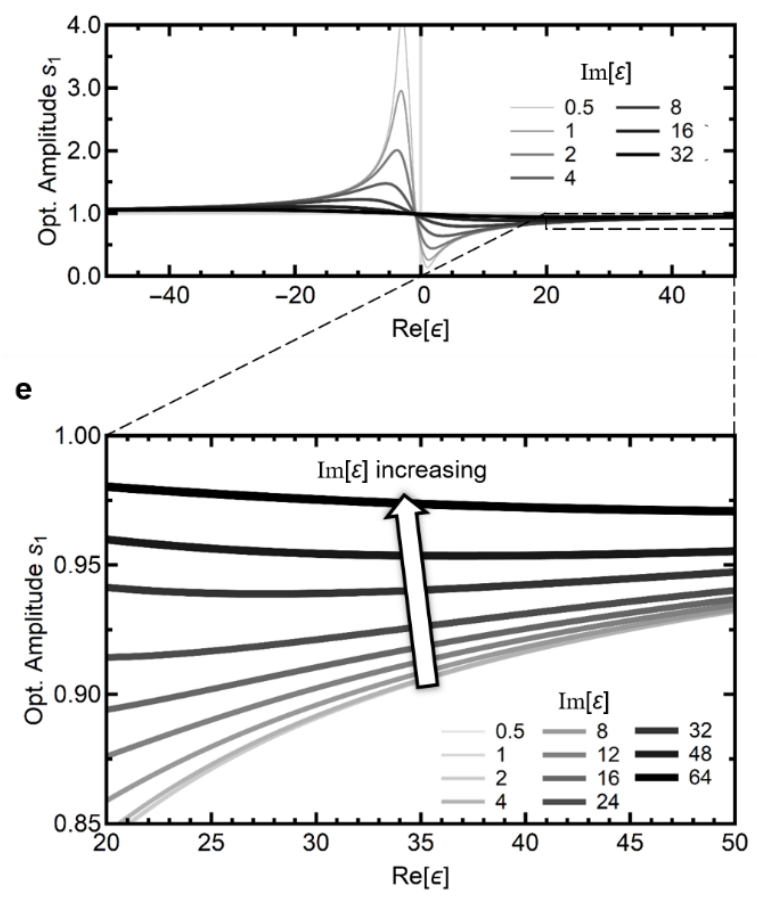

d

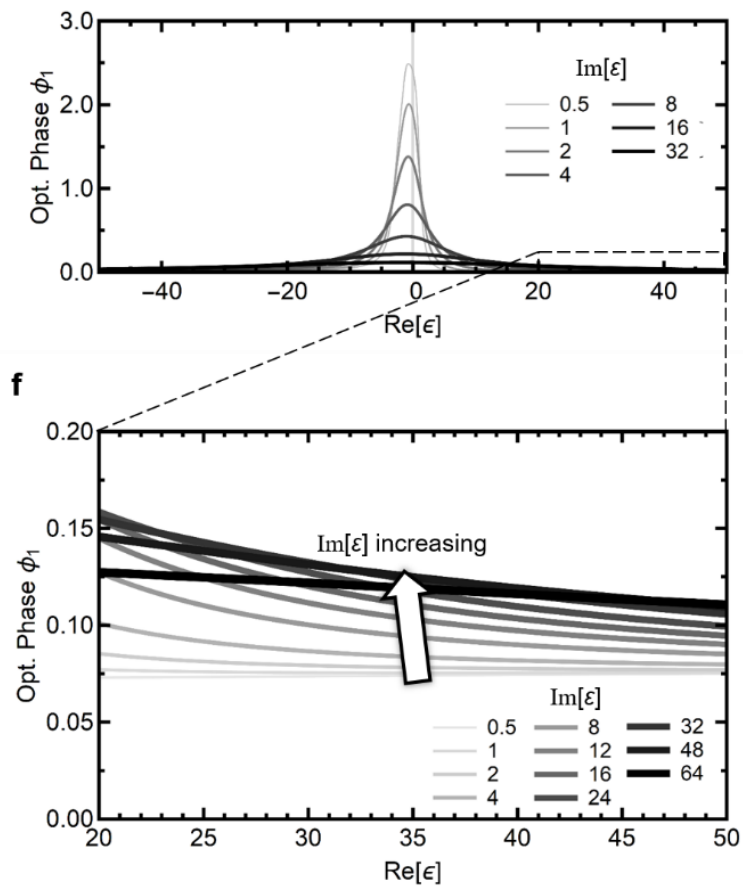

Figure S9. Dependence of near-field signal on charge carrier concentration $n$ in the regime of high $\operatorname{Re}[\varepsilon]$. a) Formulas for the dielectric function of crystalline GST326, with the real and imaginary part of the Drude contribution written explicitly and marked by curly brackets. The formulas are color-coded to explain the barely changing real part and the strongly changing imaginary part (see text for discussion). b) resulting dielectric functions (real (blue) and imaginary part (red)) for different $n$ in crystalline GST326 $\left(\mu=0.9 \mathrm{~cm}^{2} / \mathrm{Vs}\right.$ and $m^{*}=0.3 m_{0}$ ), with arrows indicating the change introduced by increasing $\left.n . \mathbf{c}\right), \mathbf{d}$ ) Coupling behaviour of the tipsample system in the Finite Dipole Model, showing the dependence of scattering amplitude (c) and phase (d) in dependence of the sample dielectric function $\varepsilon$, with the $\mathrm{x}$-axis showing $\operatorname{Re}[\varepsilon]$ and the evolution of the curves (increasing thickness) showing the influence of rising $\operatorname{Im}[\varepsilon]$ from 0.5 to 64 , leading to a dampened near-field resonance. e), f) Zoom-ins focused on the range that is relevant for crystalline GST326, around $\operatorname{Re}[\varepsilon] \approx 35$. The influence of increasing $\operatorname{Im}[\varepsilon]$ is indicated by white arrows. 


\section{TEM analysis}

Figures S10 and S11 present the results of transmission electron microscopy (TEM) analysis of RC and RA spots, respectively. Here, Figure S10 shows that two distinctive crystalline areas can be separated, with smaller crystallites in the $\mathbf{R C}$ spot and larger, column-like crystallites in the remaining $\mathbf{C}$ line (cf. Figure $\mathbf{S} 2$ for an explanation of the different regions). Figure S11 shows different TEM-EDX (energy-dispersive x-ray spectroscopy) profiles of an RA spot, to investigate possible differences in stoichiometry between $\mathbf{A}$ and $\mathbf{R A}$ regions, that could lead to the pronounced Kelvin probe contrast described in the main text. However, no changes in stoichiometry, e.g. due to Te evaporation or oxidation during the melting process, could be observed for $\mathrm{Ge}$ (yellow), $\mathrm{Sb}$ (orange), Te (dark orange) or $\mathrm{O}$ (blue), when comparing a) A, b) $\mathbf{R A}$, and c) $\mathbf{C}$ regions.
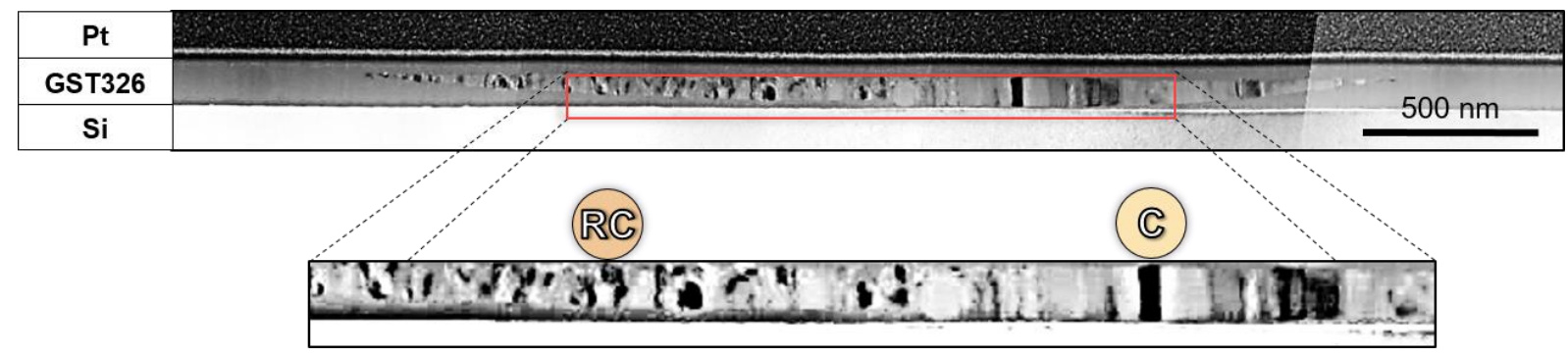

Figure S10. Top: TEM lamella cross-section of a recrystallized (RC) spot, showing the Si substrate (bottom), GST326 layer (center) and Pt covering layer (top). In the GST326 layer, two different regions are immediately distinguishable: a homogeneously bright amorphous region and a crystallized area with inhomogeneous brightness (crystallites). The crystallized area reaches down to the substrate in the center with the thickness decreasing towards the sides. Bottom: zoom-in of the recrystallized spot showing that the crystalline area can be further separated into two different regions: on the left side, the crystallites are smaller and irregularly shaped (RC); on the right side, the crystallites are larger and vertically aligned $(\mathbf{C})$, i.e. they reach from top to bottom.

a

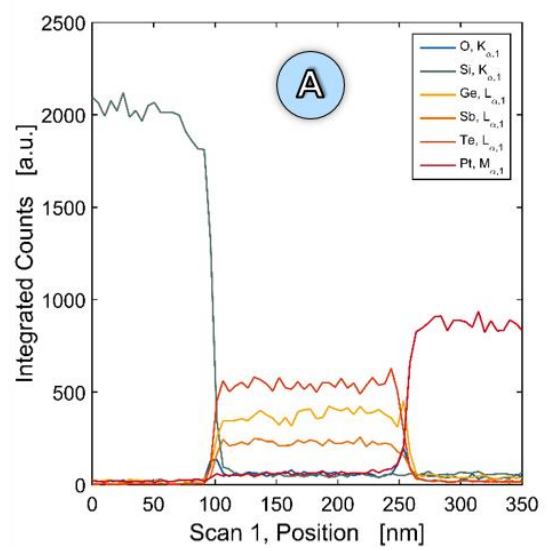

b

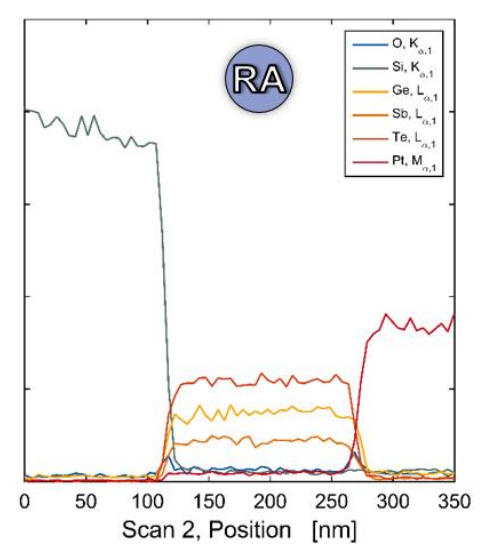

C

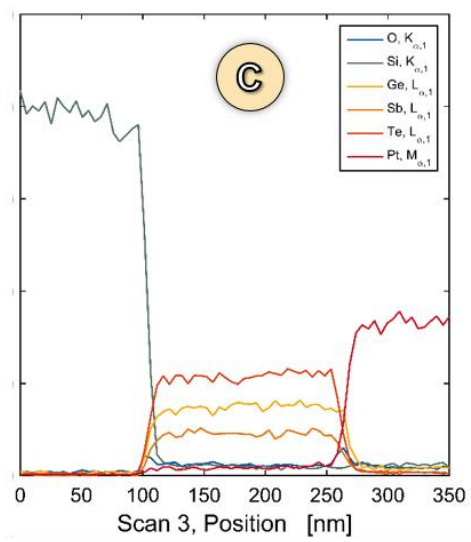

Figure S11. TEM-EDX profiles of GST326 lamella through a reamorphized (RA) spot at three different positions of the TEM lamella: a) as-deposited amorphous region $\mathbf{A}$ outside the spot, b) reamorphized region $\mathbf{R A}$ inside the spot, and c) crystalline region $\mathbf{C}$ next to the spot. 


\section{References}

(1) Michel, A.-K. U.; Heßler, A.; Meyer, S.; Pries, J.; Yu, Y.; Kalix, T.; Lewin, M.; Hanss, J.; Rose, A. de; Maß, T. W. W.; Wuttig, M.; Chigrin, D. N.; Taubner, T. Advanced Optical Programming of Individual Meta-Atoms Beyond the Effective Medium Approach. $A d v$. Mater. 2019, 31 (29), 1901033. DOI: 10.1002/adma.201901033.

(2) Heßler, A.; Wahl, S.; Leuteritz, T.; Antonopoulos, A.; Stergianou, C.; Schön, C.-F.; Naumann, L.; Eicker, N.; Lewin, M.; Maß, T. W. W.; Wuttig, M.; Linden, S.; Taubner, T. $\mathrm{In}_{3} \mathrm{SbTe}_{2}$ as a programmable nanophotonics material platform for the infrared. Nat. Commun. 2021, 12, 924. DOI: 10.1038/s41467-021-21175-7.

(3) Klopf, J. M.; Helm, M.; Kehr, S. C.; Lehnert, U.; Michell, P.; Pashkin, A.; Schneider, H.; Seide, W.; Winnerl, S.; Zvyagin, S. FELBE-Upgrades and Status of the IRLTHZ FEL User Facility at HZDR. 43rd International Conference on Infrared, Millimeter, and Terahertz. Waves (IRMMW-THz) 2018, 520-521.

(4) Knoll, B.; Keilmann, F. Enhanced dielectric contrast in scattering-type scanning nearfield optical microscopy. Opt. Commun. 2000, 182 (4-6), 312-328. DOI: 10.1016/S00304018(00)00826-9.

(5) Lang, D.; Döring, J.; Nörenberg, T.; Butykai, Á.; Kézsmárki, I.; Schneider, H.; Winnerl, S.; Helm, M.; Kehr, S. C.; Eng, L. M. Infrared nanoscopy down to liquid helium temperatures. Rev. Sci. Instrum. 2018, 89 (3), 33702. DOI: 10.1063/1.5016281.

(6) Wurtz, G.; Bachelot, R.; Royer, P. A reflection-mode apertureless scanning near-field optical microscope developed from a commercial scanning probe microscope. Rev. Sci. Instrum. 1998, 69 (4), 1735-1743. DOI: 10.1063/1.1148834.

(7) Nonnenmacher, M.; O’Boyle, M. P.; Wickramasinghe, H. K. Kelvin probe force microscopy. Appl. Phys. Lett. 1991, 58 (25), 2921-2923. DOI: 10.1063/1.105227.

(8) Zerweck, U.; Loppacher, C.; Otto, T.; Grafström, S.; Eng, L. M. Accuracy and resolution limits of Kelvin probe force microscopy. Phys. Rev. B 2005, 71 (12), 125424. DOI: 10.1103/PhysRevB.71.125424.

(9) Nörenberg, T.; Wehmeier, L.; Lang, D.; Kehr, S. C.; Eng, L. M. Compensating for artifacts in scanning near-field optical microscopy due to electrostatics. APL Photonics 2021, 6 (3), 36102. DOI: 10.1063/5.0031395.

(10) Meyer, S.; Tan, Z. Y.; Chigrin, D. N. Multiphysics simulations of adaptive metasurfaces at the meta-atom length scale. Nanophotonics 2020, 9 (3), 675-681. DOI: 10.1515/nanoph2019-0458.

(11) Gucciardi, P. G.; Bachelier, G.; Allegrini, M. Far-field background suppression in tipmodulated apertureless near-field optical microscopy. J. Appl. Phys. 2006, 99 (12), 124309. DOI: $10.1063 / 1.2208527$.

(12) Moon, K.; Park, H.; Kim, J.; Do, Y.; Lee, S.; Lee, G.; Kang, H.; Han, H. Subsurface nanoimaging by broadband terahertz pulse near-field microscopy. Nano Lett. 2015, 15 (1), 549-552. DOI: 10.1021/n1503998v.

(13) Sternbach, A. J.; Hinton, J.; Slusar, T.; McLeod, A. S.; Liu, M. K.; Frenzel, A.; Wagner, M.; Iraheta, R.; Keilmann, F.; Leitenstorfer, A.; Fogler, M.; Kim, H.-T.; Averitt, R. D.; 
Basov, D. N. Artifact free time resolved near-field spectroscopy. Opt. Express 2017, 25 (23), 28589-28611. DOI: 10.1364/OE.25.028589.

(14) Jost, P.; Volker, H.; Poitz, A.; Poltorak, C.; Zalden, P.; Schäfer, T.; Lange, F. R. L.; Schmidt, R. M.; Holländer, B.; Wirtssohn, M. R.; Wuttig, M. Disorder-Induced Localization in Crystalline Pseudo-Binary GeTe-Sb $\mathrm{Te}_{3}$ Alloys between $\mathrm{Ge}_{3} \mathrm{Sb}_{2} \mathrm{Te}_{6}$ and GeTe. Adv. Funct. Mater. 2015, 25 (40), 6399-6406. DOI: 10.1002/adfm.201500848.

(15) Wuttig, M.; Deringer, V. L.; Gonze, X.; Bichara, C.; Raty, J.-Y. Incipient Metals: Functional Materials with a Unique Bonding Mechanism. Adv. Mater. 2018, 30 (51), 1803777. DOI: 10.1002/adma.201803777.

(16) Kremers, S. Optische Eigenschaften von Phasenwechselmaterialien für zukünftige optische und elektronische Speicheranwendungen, RWTH Aachen, 2009.

(17) Cvitkovic, A.; Ocelic, N.; Hillenbrand, R. Analytical model for quantitative prediction ofmaterial contrasts in scattering-type near-field optical microscopy. Opt. Express 2007, 15 (14), 8550-8565. DOI: 10.1364/OE.15.008550.

(18) Hauer, B.; Engelhardt, A. P.; Taubner, T. Quasi-analytical model for scattering infrared near-field microscopy on layered systems. Opt. Express 2012, 20 (12), 13173-13188. DOI: 10.1364/OE.20.013173.

(19) Ocelic, N. Quantitative Near-field Phonon-polariton Spectroscopy, TU München, 2007.

(20) Hauer, B. Nano-optical mapping of permittivity contrasts and electronic properties at the surface and beneath, RWTH Aachen, 2015.

(21) Taubner, T.; Hillenbrand, R.; Keilmann, F. Performance of visible and mid-infrared scattering-type near-field optical microscopes. J. Microsc. 2003, 210 (3), 311-314. DOI: 10.1046/j.1365-2818.2003.01164.x.

(22) Keilmann, F.; Hillenbrand, R. Near-field microscopy by elastic light scattering from a tip. Phil. Trans. R. Soc. Lond. A 2004, 362 (1817), 787-805. DOI: 10.1098/rsta.2003.1347. 